\title{
Monocyte Chemoattractant Protein-1 in Human Atheromatous Plaques
}

Nicolas A. Nelken," Shaun R. Coughlin, " David Gordon," and Josiah N. Wilcox"

Cardiovascular Research Institute and ${ }^{*}$ Division of Vascular Surgery and ${ }^{\ddagger}$ Department of Medicine, University of California, San Francisco, San Francisco, California 94143; ' University of Washington, Department of Pathology, Seattle,

Washington 98195; and "Genentech Corporation, South San Francisco, California 94080

\begin{abstract}
Monocytes appear to be central to atherogenesis both as the progenitors of foam cells and as a potential source of growth factors mediating intimal hyperplasia, but the chemical messages which stimulate the influx of monocytes into human atheroma remain unknown. Monocyte chemoattractant protein-1 (MCP-1) is a recently described molecule with powerful monocyte chemotactic activity expressed by monocytes, vascular endothelial cells, and smooth muscle cells in culture. To begin to address the role of MCP-1 in vivo, we examined 10 normal arteries and 14 diseased human arteries for MCP-1 expression by in situ hybridization. MCP-1 mRNA was detected in $16 \%$ of 10,768 cells counted in human carotid endarterectomy specimens with highest expression seen in organizing thrombi (33\%) and in macrophage rich areas bordering the necrotic lipid core (24\%) as compared to the fibrous cap (8\%) and the necrotic lipid core itself (5\%). Based on immunohistochemical staining of serial sections and on cell morphology, MCP-1 mRNA appeared to be expressed by vascular smooth muscle cells (VSMC), mesenchymal appearing intimal cells (MICs), and macrophages. By contrast, few cells expressing MCP-1 mRNA were found in normal arteries $(<0.1 \%)$. These data suggest a potential role for MCP-1 in mediating monocytic infiltration of the artery wall. (J. Clin. Invest. 1991. 88:1121-1127.) Key words: In situ hybridization • vascular smooth muscle cells • vascular disease • macrophages $\cdot$ cytokines
\end{abstract}

\section{Introduction}

Monocyte-derived foam cells are a major component of atheroma, comprising as much as $60 \%$ of cells found in the necrotic lipid core, and $10-20 \%$ of cells in the fibrous cap (1). These cells have been demonstrated not only in mature plaques but also in early fatty streaks in animal models of atherogenesis and in human disease (2). It has been suggested that growth factors produced by plaque macrophages may be important in terms of plaque progression (3). The signals which attract monocytes

Reprint requests should be addressed to Josiah N. Wilcox, Division of Hematology/Oncology, Emory University School of Medicine, 308 Woodruff Memorial Building, Drawer AR, Atlanta, GA 30322.

Received for publication 8 November 1990 and in revised form 16 April 1991.

1. Abbreviations used in this paper: MCP-1, monocyte chemoattractant protein-1; MIC, mesenchymal intimal cells; $P C R$, polymerase chain reaction; VSMC, vascular smooth muscle cell.

J. Clin. Invest.

(c) The American Society for Clinical Investigation, Inc.

0021-9738/91/10/1121/07 $\$ 2.00$

Volume 88, October 1991, 1121-1127 into these lesions remain unknown. Monocyte chemoattractant protein-1 (MCP-1) ${ }^{1}$ is a secreted molecule with potent chemotactic activity for monocytes (4). MCP-1 is expressed by cultured glioma cells (4), fibroblasts (5), monocytes (6), lymphocytes (7), endothelial cells (8), and smooth muscle cells (9). $\operatorname{TNF} \alpha(5,8,10), \operatorname{IL}-1 \beta(5,8,10), \gamma \operatorname{IFN}(8)$, and endotoxin $(5$, 10) have all been shown to increase MCP-1 mRNA levels in cultured endothelial cells and fibroblasts. In addition, increases in MCP-1-like activity and MCP-1 mRNA have been documented in supernatants of human umbilical vein endothelial cells (HUVE's) stimulated with serum enriched with minimally oxidized LDL and $\beta$ VLDL $(11,12)$. These in vitro studies suggest a potential role for MCP-1 in the recruitment of monocytes into the vessel wall in the setting of inflammation or hyperlipidemia.

To address whether MCP-1 may play a role in recruiting monocytes to the vessel wall in vivo, we set out to identify MCP-1 mRNA production in human atheroma using in situ hybridization. Atherosclerotic plaques exhibited marked hybridization, especially in macrophage-rich inflammatory regions. By comparison, minimal expression was noted in control arteries without atherosclerotic disease.

To identify possible regulators of MCP-1 production in cells derived from human atheroma, vascular smooth muscle cells (VSMCs) grown from human carotid endarterectomy specimens were stimulated with various cytokines and MCP-1 mRNA levels were determined by Northern blot. Tumor necrosis factor (TNF $\alpha$ ), gamma interferon ( $\gamma$ IFN), and supernatants from mixed lymphocyte culture were shown to stimulate production of MCP-1 mRNA, whereas $10 \%$ FCS did not.

These data suggest a potential in vivo role for MCP-1 in mediating monocytic infiltration of the artery wall, and a role for induction of MCP-1 expression by inflammatory mediators in modulating this process.

\section{Methods}

MCP-1 probe isolation. The CDNA for MCP-1 was cloned from stimulated human lung fibroblasts using polymerase chain reaction (PCR) amplification of cDNA made from cytoplasmic RNA. Human lung fibroblasts were obtained from explants obtained at autopsy. Cultures were maintained in DMEM supplemented with $10 \%$ FCS, $1 \%$ penicillin/streptomycin, and $25 \mathrm{mM}$ Hepes, buffer pH 7.4. To increase MCP1 mRNA levels, cells were stimulated with phorbol myristic acetate $(100 \mathrm{ng} / \mathrm{ml}$ for $48 \mathrm{~h})$, then with $E$. coli endotoxin $(10 \mu \mathrm{g} / \mathrm{ml}$, Sigma Corp., St. Louis, MO) in the presence of $1 \mu \mathrm{g} / \mathrm{ml}$ cycloheximide (Sigma Chemical Corp.) for $6 \mathrm{~h}$ (13). Total RNA was isolated using guanidium thiocyanate lysis and $\mathrm{CsCl}$ density gradient centrifugation (14). cDNA was then produced using 12-18mer oligo $d T$ primers (Pharmacia Fine Chemicals, Piscataway, NJ) and reverse transcription followed by RNAse A digestion. 30 cycles of PCR were performed (15) using 33mer oligonucleotide primers which primed the $5^{\prime}$ and $3^{\prime}$ ends of the coding region for MCP-1 and encoded protruding Xho I ( 5 ' primer) and Eco RI (3' primer) restriction sites. The PCR product was subcloned into 

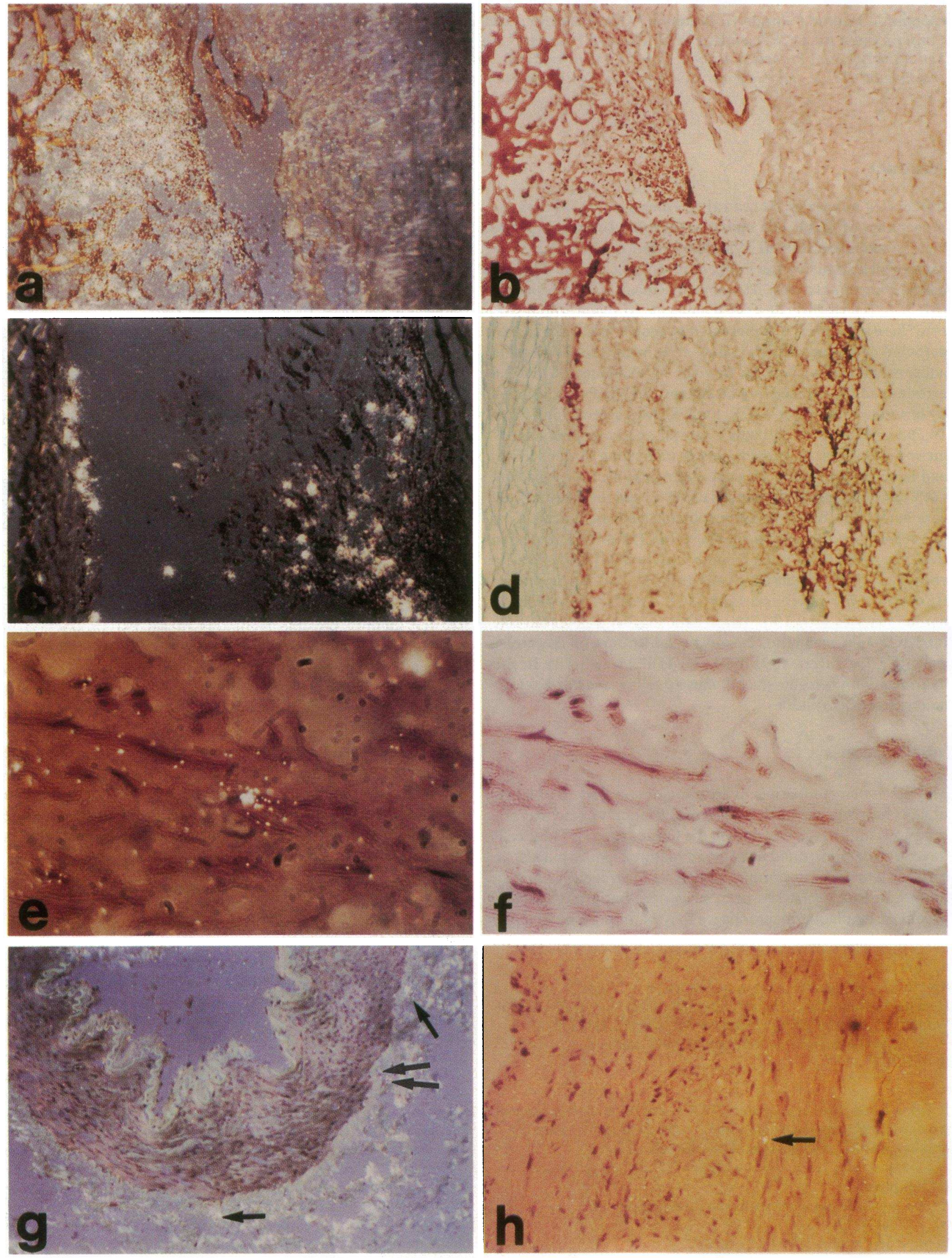
the Xho I and Eco RI sites of Bluescript SK-plasmid and its sequence determined by dideoxy nucleotide sequencing (16). Primer sequences were $5 '$ CCC CTC GAG TCC AGC ATG AAA GTC TCT GCC CTT for the $5^{\prime}$ sequence, and 5' CCC GAA TTC TGT TCA AGT CTT CGG AGT TTG GGT for the $3^{\prime}$ complementary sequence.

In situ hybridization. Human carotid endarterectomy specimens were obtained directly from the operating room, fixed for $2 \mathrm{~h}$ at $4^{\circ} \mathrm{C}$ in $4 \%$ paraformaldehyde immediately upon surgical excision, cryopreserved in $15 \%$ sucrose for $3 \mathrm{~h}$ and frozen in OCT at $-70^{\circ} \mathrm{C}$. To construct riboprobes, Bluescript $\mathrm{KS}+$ plasmid containing a full-length MCP-1 insert was made linear by restriction digestion with Eco RI or Xho I (for sense and antisense probes, respectively), then transcribed using T3 or T7 RNA polymerase (Stratagene Corp., La Jolla, CA) in the presence of ${ }^{35} \mathrm{~S}$-UTP. In situ hybridization was performed on $10 \mu \mathrm{m}$ frozen histologic sections as previously described $(17,18)$. After coating with photographic emulsion, sections were exposed for 4-12 wk. Exposed sections were counterstained with hematoxylin and eosin.

The proportion of cells expressing MCP-1 was determined by counting 10,768 individual cells identified by nuclear staining at $400 \times$ in the 14 atherosclerotic specimens hybridized to the MCP-1 riboprobe (Fig. 1, $e$ and $f$ ). A cell was considered positive if the number of silver grains associated with stained nuclei exceeded five times background over equivalent cell-sized areas without nuclei. For most sections we screened, cells with seven or more grains were considered positive.

Immunohistochemistry. Serial microscopic frozen sections immediately adjacent to sections hybridized with the MCP-1 probe were used for immunohistochemical identification of cells in three atherosclerotic specimens. Primary monoclonal antibodies included HAM56 (Dako Corp., Carpinteria, CA) which reacts with 95\% of the human monocyte/macrophage cell population (19). Cross-reactivity of this antibody has been shown to capillary endothelium, hence, all slides stained with HAM56 were compared with sequential slides stained with Ulex europaeus lectin (Vector Laboratories, Inc., Burlingame, CA), which selectively stains endothelium (20). Vascular smooth muscle cells were identified with HHF35 (Enzo Pharmaceuticals, New York, NY) to muscle actin which specifically reacts with cardiac, skeletal, and smooth muscle cells, but not fibroblasts, endothelium, neural tissue, or epithelium (21). T lymphocytes were identified with UCHL1 (Dako Corp., Santa Barbara, CA) which reacts with CD45RO expressed by prestimulated and a subset of resting $T$ cells (22). This antibody does not react with B cells or NK cells, although there is some cross-reactivity with cells of myeloid and monocytic lineage (PMNs were rare in these specimens). Appropriate biotin-conjugated secondary antibodies were used and detected with avidin-alkaline phosphatase (ABC-AP kit; Vector Laboratories, Inc.) according to the manufac- turer's instructions as reported previously $(17,18)$. Counterstaining was done with $0.4 \%$ methyl green (Sigma Corp.).

Cell culture. VSMCs were cultured in collagen matrix (Vitrogen; Collagen Corp., Palo Alto, CA) from human atheroma obtained at carotid endarterectomy using published techniques (23). Medium was changed twice weekly, and cells passed before reaching confluence. All cells were used within the first six passages.

Smooth muscle cell phenotype was confirmed by immunohistochemical staining using HHF35 and fluorescein secondary antibody. Cultured cells stained positive for actin in a spiculated pattern typical for smooth muscle cells in $83 \pm 5 \%$ (95\% confidence interval) of 195 cells counted (not shown).

Northern blotting. VSMCs grown to $90 \%$ confluence in $150 \mathrm{~cm}^{2}$ flasks were made quiescent by incubating for $48 \mathrm{~h}$ with serum-free medium after washing $3 \times$ with PBS. Thereafter, they were either stimulated for 4 or $24 \mathrm{~h}$ with the cytokines or growth factors used in each specific experiment. Total cellular RNA was isolated using the guanidium isothiocyanate method (14). $10 \mu \mathrm{g}$ of glyoxal-treated RNA was then run in a $1 \%$ agarose gel and transfered to nylon reinforced nitrocellulose (Schleicher \& Schuell Corp., Keene, NH) as described (14). ${ }^{32} \mathrm{P}$-labeled MCP-1 probe was produced using the random primer technique and T7 DNA polymerase (Stratagene Random Primer kit; Stratagene Corp., La Jolla, CA). Nitrocellulose membranes were prehybridized for $4 \mathrm{~h}$ and hybridized at $42^{\circ}$ over night at a concentration of $10^{6}$ $\mathrm{cpm} / \mathrm{ml}$ in buffer containing $50 \%$ formamide. Two washes using $2 \times$ SSC with $0.1 \%$ SDS at $55^{\circ}$ and two high-stringency washes using $0.1 \times$ SSC with $0.1 \%$ SDS at $55^{\circ}$ were performed for 15 min each. Nitrocellulose was then exposed to Kodak X-OMAT film with an intensifying screen at $-70^{\circ}$ for variable periods of time depending on estimated radioactivity of the nitrocellulose membranes.

\section{Results}

In situ hybridization. 14 carotid endarterectomy specimens, eight normal coronary arteries, and two normal internal mammary arteries were examined by in situ hybridization. Positive hybridization for MCP-1 was clearly present in all atheroma examined (Fig. 1, $a, c, e$ ). No cellular hybridization was seen in control slides probed with MCP-1 sense riboprobe (data not shown).

Expression of MCP-1 in histologically defined regions of human atheroma. Positive hybridization was detected in $16 \%$ of 10,768 cells counted. Random high-power fields among representative histologic regions were scored using a counting grid

Figure 1. (a) A portion of atherosclerotic plaque showing extensive hybridization of MCP-1 antisense probe at 8 wk exposure. Area of inflammatory cell infiltrate in organizing thrombus hybridizes most strongly in this section. Darkfield polarized light epiluminescence view enhances silver grains corresponding to areas of hybridization (8-wk exposure; magnification, 100). (b) Same field as $a$ in brightfield stained with hematoxylin and eosin showing fibrous cap area (right) adjacent to organizing thrombus in intraplaque hemorrhage (left). Infiltration of inflammatory cells can be seen between these two areas (magnification, 100). (c) Darkfield polarized-light epiluminescence view of carotid atherosclerotic plaque showing hybridization for MCP-1 in the inflammatory cell-rich area underlying the necrotic lipid core abutting the arterial media (above) (8-wk exposure; magnification, 100). (d) Adjacent frozen section to $c$ stained with antimacrophage monoclonal antibody (HAM56) showing correlation of immunohistochemical staining for macrophages with areas of intense hybridization seen in $c$. Positive staining appears red compared with methyl green counterstain (magnification, 100). (e) High-power darkfield polarized light epiluminescence view of an atherosclerotic plaque showing MCP-1 hybridization in a VSMC in the fibrous cap region. Discrete vascular smooth muscle cells were shown to hybridize strongly and were often surrounded by morphologically similar cells which did not hybridize (8-wk exposure, magnification, 500$)$. $(f)$ Same field as $e$ in lightfield view for comparison, showing an area with a high percentage of smooth muscle cells (positive HHF35 staining on serial section not shown). Note typical elongated nucleus and smooth muscle morphology in hybridized cell seen in $e$. (g) Section of normal internal mammary artery obtained from coronary bypass surgical specimens hybridized with MCP-1 antisense probe. Very low levels of hybridization were seen in normal vessels. Examination of this section under high power revealed only four cells hybridizing to MCP-1 probe (arrows). The other bright areas in the adventitia represent refractile fibrous tissue, a normal feature of the adventitia when viewed by epiluminescence (8-wk exposure; magnification, 100). (h) Section of control coronary artery obtained from cardiomyopathy patient during heart transplantation (native heart) hybridized with MCP-1 antisense probe. Hybridization was rare in these specimens. One smooth muscle-appearing cell can be seen in the media with higher than background hybridization (arrow). Concentric fibrous intimal thickening was a universal finding in adult coronary specimens, however, it was not associated with hybridization of probe (8-wk exposure; magnification, 125). 

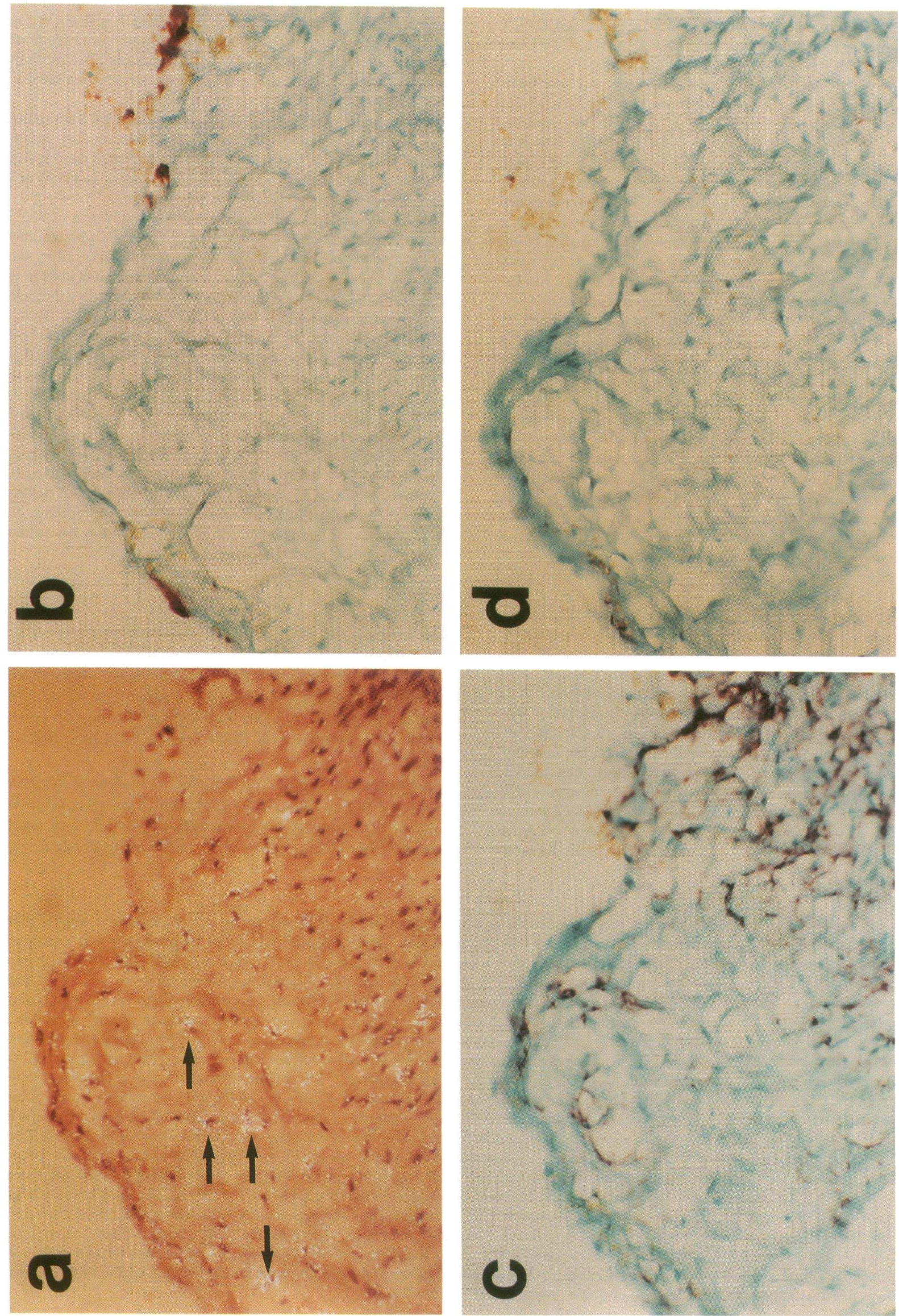
Table I. Comparison of Percentage Nuclei Associated with In Situ Hybridization to MCP-1 $\mathrm{mRNA}$

Positive cells/total cells
Morphologic subdivision

Fibrous cap (uniform portion)

Fibrous cap (pleomorphic portion)

Inflammatory area underlying necrotic core

Necrotic core

Organizing thrombus

Immunohistochemical subdivisic

HAM56 positive cells

HHF35 positive cells

Total
$76 / 1471(5 \%)$

288/2871 (10\%)

$482 / 1999(24 \%)$

$28 / 475(6 \%)$

$439 / 1163(38 \%)$

$82 / 1754(5 \%)$

$1737 / 10768(16 \%)$
$342 / 1035(33 \%)$

A cell was considered positive if the number of silver grains associated with stained nuclei exceeded five times background over equivalent cell-sized areas. For most sections we screened, cells with seven or more grains were considered positive. Both morphologic and immunohistochemical criteria were used for subdivision of tissues. For the morphologic subdivisions, uniform and pleomorphic portions of the fibrous cap were seen to have different morphologic features and to hybridize to different degrees (see text for details), and were separated on those grounds. For immunohistochemical subdivisions, HAM56 identified cells of monocyte lineage (primarily macrophages), and HHF35 identified VSMCs. Results show a preponderance of hybridization to macrophages, inflammatory areas underlying the necrotic lipid core, and organizing thrombus. Vascular smooth muscle, however, was also seen to hybridize but to a lesser degree.

and the percentage of hybridized cells tallied (Table I). For the purposes of this analysis we categorized areas of the 14 atherosclerotic plaques into five histologically identifiable regions: $(a)$ fibrous cap containing uniform elongated "pancake" nuclei arranged in a primarily unidirectional orientation, $(b)$ fibrous cap containing pleomorphic nuclei without ordered orientation, (c) inflammatory areas bordering the necrotic lipid core, (d) necrotic lipid core, characterized by cellular debris, and cholesterol clefts, and $(e)$ organizing intraplaque thrombus characterized by the presence of morphologically identifiable fibrin deposits or hemosiderin containing macrophages.

Fibrous cap. In the fibrous cap, $8 \%$ of 4,342 cells hybridized to the MCP-1 riboprobe (Fig. $1, e$ and $f$ ). Significantly more MCP-1-positive cells were found in areas of the fibrous cap regions predominantly containing cells with pleomorphic nuclei arranged in a random orientation ( $10 \%$ of 2,871 cells) compared with fibrous cap regions containing predominantly uniform spindle shaped cells typical of VSMCs ( $5 \%$ of 1,471 cells, $P<0.001$, chi square). The in situ signal intensity was generally lower over cells in the fibrous cap compared with the intense signals seen in the same sections most notably in inflammatory areas bordering the necrotic lipid core (see below).
Regions of the fibrous cap with uniform spindle-shaped cells generally showed uniform staining with the HHF35 antibody. Fibrous cap regions with pleomorphic nuclei often exhibited fewer VSMCs staining with HHF35 and frequently contained HAM56 and a few UCHL1-positive cells as well. MCP1-positive cells in the fibrous cap included a population of mesenchymal appearing intimal cells (MIC) often in pleomorphic zones which did not co-stain with any of the antibodies used in this study (Fig. 2). These cells had large pale nuclei distinct from the typical spindle-shaped smooth muscle cell nucleus, a stellate cytoplasm, and were morphologically similar to the platelet-derived growth factor (17) or tissue factor (18) mRNA-containing MICs described previously in atherosclerotic plaques. Many areas within the atherosclerotic plaques examined contained numerous cells meeting these criteria especially in the pleomorphic regions of the fibrous cap and areas of organizing thrombi. It is difficult to estimate the proportion of positive MICs because their detection is by a process of exclusion rather than direct identification.

Organizing thrombus. Many cells hybridized strongly to the MCP-1 probe in areas of focal organizing thrombi (33\% of 1,035 cells; Fig. 1, $a$ and $b$ ). These regions often contained many HAM56-positive cells with some UCHL1-positive T cells. Spatial correlation of HAM56-positive cells and cells hybridizing for MCP-1 strongly suggests that macrophages express MCP-1. We cannot exclude the possibility that some expression in these areas is by MICs because these cells are often found in areas of focal organizing thrombus $(24,25)$.

Necrotic lipid core. Discrete cells in the necrotic lipid core were markedly positive for MCP-1 mRNA (6\% of 475 cells) and were often adjacent to nonhybridizing cells. The necrotic cores exhibited diminished cellular density and contained predominantly necrotic debris, foam cell deposits, cholesterol clefts, and calcification. In agreement with previous studies macrophages were the predominant cell type found in the necrotic core although UCHL1-positive cells were also identified in these regions (1).

Inflammatory regions bordering the necrotic lipid core. Of all the regions studied the most intensely hybridizing MCP-1-positive cells were found in inflammatory regions bordering the necrotic core. Positive cells were relatively abundant, representing $24 \%$ of 1,999 cells counted. These inflammatory regions contained many HAM56-positive cells with a few scattered UCHL1-positive cells. Histologically identified foam cells were often observed to hybridize to the MCP-1 probe in these areas.

Expression of $M C P-1$ by cell type. To identify specific cell types associated with MCP-1 production, serial microscopic sections immediately adjacent to sections hybridized with the MCP-1 probe were used for immunohistochemical staining with HAM56, UCHL1, HHF35, and Ulex lectin in three representative specimens. Carefully chosen regions which stained exclusively with HAM56 were found to be strongly positive for

Figure 2. (a) Brightfield epiluminescent view of cellular area with a high proportion of immunohistochemically unstained mesenchymal appearing intimal cells (MICs) in carotid atherosclerotic plaque (arrows). Nuclei are visible as the dark hematoxylin-stained bodies surrounded by bright silver grains. Hematoxylin and eosin section; magnification, 200. (b) Adjacent section to $a$ stained with HAM56, showing lack of tissue macrophages in this section (typical cross-reactivity to endothelium by this antibody is also illustrated). (c) Serial section stained with HHF35 for actin-containing VSMCs. Some positive cells were noted, but do not appear to be related to those hybridizing for MCP-1 in this section. (d) Serial section stained with UCHL1 showing absence of $\mathrm{T}$ cells in this section. Positive staining for each monoclonal antibody appears red, compared with methyl green counterstaining. Lack of immunohistochemical staining in areas of marked hybridization indicates the presence of MICs. 
MCP-1 (38\% of 1,754 cells). Cells meeting histologic criteria for macrophages, including large pale nucei with foamy cytoplasm, clearly hybridized with MCP-1 riboprobe, whereas hemosiderin containing macrophages did not. Fewer MCP-1 positive cells were found in regions staining exclusively with HHF35 (5\% of 1,163 cells). These areas showed no HAM56 staining. Despite the low percentage of cells hybridizing in these areas, individual cells clearly meeting histologic criteria for VSMCs, including spindle-shaped nuclei and elongated cytoplasm, hybridized for MCP-1 (Fig. 1, $e$ and $f$ ). It is not possible to comment on the colocalization of $\mathrm{T}$ cell staining with MCP-1 hybridization because no region was found to stain exclusively with UCHL1 in the absence of HAM56 staining. These data suggest that both VSMCs and macrophages can express MCP-1 in vivo. In addition, cells not staining with HAM56 or HHF35, meeting the histological criteria for MICs were also seen to hybridize strongly for MCP-1.

We failed to detect MCP-1 hybridization to endothelial cells lining the vasa vasorum or on the lumenal surface of the plaques. Given the fact that the total amount of endothelium seen in these specimens was small due to transverse sectioning, lack of endothelium in portions of the plaque and in some cases loss of endothelium in preparation, conclusions regarding $\mathrm{MCP}-1$ expressed by the endothelium in vivo cannot be drawn from this study.

Normal, nondiseased internal mammary artery specimens obtained from coronary bypass operations exhibited little production of MCP-1 mRNA as determined by in situ hybridization. Hybridizing cells, if present, tended to be found in the adventitia (Fig. $1 \mathrm{~g}$ ). Occasional macrophage staining was seen in the adventitia suggesting that hybridizing cells may be scattered tissue macrophages. Like the internal mammary arteries, normal coronary vessels obtained from explanted hearts also showed only minimal MCP-1 expression (Fig. $1 h$ ). These specimens were obtained from nonatherosclerotic coronary arteries in patients with idiopathic cardiomyopathies undergoing heart transplantation. A small amount of concentric intimal thickening which was invariably present in these specimens but no advanced atherosclerotic lesions were seen. Hybridization was found in $<0.1 \%$ of cells counted in normal vessels.

Cytokine stimulation of VSMCs in culture. The finding that VSMCs expressed MCP-1 in vivo suggested a possible role for smooth muscle-produced MCP-1 in initiating or enhancing monocytic infiltration of the vessel wall. To begin to examine factors potentially responsible for regulation of MCP-1 expression by VSMCs, Northern blot analysis was performed on cytokine-treated human VSMCs in culture. Northern blot analysis of $10 \mu \mathrm{g}$ of total cellular RNA per sample revealed a very low level of detectable MCP-1 RNA in resting cells as well as those stimulated with $10 \%$ FCS. Supernatants from a mixed lymphocyte culture (donated by Bruce Hall, Stanford University, Palo Alto, CA) however, produced a marked increase in MCP-1 mRNA detected at 4 and $24 \mathrm{~h}$ (data not shown). Inflammatory regulators TNF $\alpha(200 \mathrm{U} / \mathrm{ml})$ and $\gamma \mathrm{IFN}(50 \mathrm{U} / \mathrm{ml})$ were then investigated alone and in the presence of $5 \%$ FCS. Both cytokines stimulated marked increases in the level of MCP-1 RNA (Fig. 3). TNF $\alpha$ elicited greater increases at $24 \mathrm{~h}$ than at $4 \mathrm{~h}$, whereas $\gamma$ IFN generated maximal response at $4 \mathrm{~h}$ with diminution over time. The differing tempos of MCP-1 induction after TNF $\alpha$ and $\gamma$ IFN stimulation presumably reflect different mechanisms of action. There was no appreciable difference in

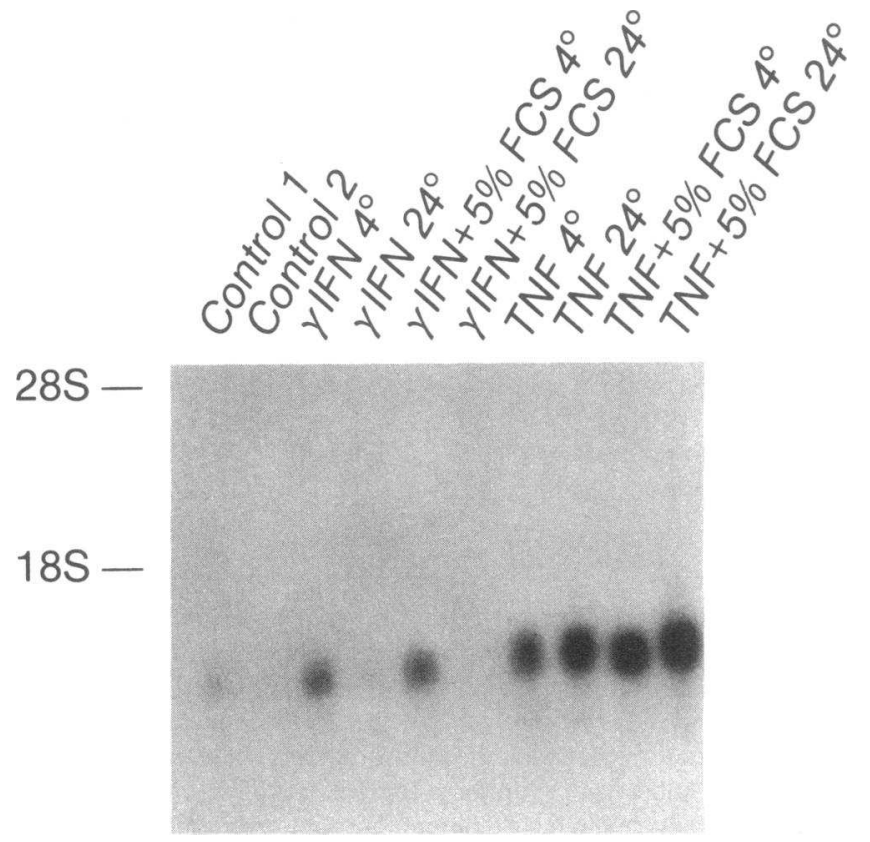

Figure 3. Northern blot of whole cell lysate RNA ( $10 \mu \mathrm{g}$ per lane) hybridized with ${ }^{32} \mathrm{P}$ labeled MCP-1 probe. Lanes include duplicate sample control cells incubated in serum-free medium for $48 \mathrm{~h} ; \gamma \mathrm{IFN}$, $50 \mathrm{U} / \mathrm{ml}$ for 4 and $24 \mathrm{~h} ; \gamma \mathrm{IFN}, 50 \mathrm{U} / \mathrm{ml}$ with $10 \%$ FCS for 4 and 24 $\mathrm{h} ; \mathrm{TNF} \alpha, 200 \mathrm{U} / \mathrm{ml}$ for 4 and $24 \mathrm{~h}$; TNF $\alpha, 200 \mathrm{U} / \mathrm{ml}$ with $10 \%$ FCS for 4 and $24 \mathrm{~h}$. Very low level baseline production is seen in control lanes compared with peaks at $4 \mathrm{~h}$ for $\gamma$ IFN and $24 \mathrm{~h}$ for TNF $\alpha$. FCS had no effect on production of MCP-1 mRNA either in cytokine treated cells (above) or otherwise unstimulated cells (not shown).

the responses to cytokines seen in the presence or absence of fetal calf serum.

\section{Discussion}

Human monocyte chemoattractant protein-1 (MCP-1) is a secreted peptide which exhibits powerful monocyte chemoattractant properties at nanomolar concentration. It has been detected in smooth muscle cells, endothelium, stimulated monocytes, lymphocytes, fibroblasts, and various tumor cell lines (6). Because the signals which attract monocytes to the atherosclerotic plaque remain unknown, we examined arteries for MCP-1 production in vivo. We have shown that there is easily detectable MCP-1 expression by multiple cell types in various regions of the atherosclerotic plaque, but little expression in normal vessels.

The majority of MCP-1 production was seen in macrophages. MCP-1 was noted in all regions of the plaque with high concentrations of macrophages, especially underlying necrotic lipid cores and in organizing thrombi. The production of MCP1 by macrophages may represent an amplification mechanism for the recruitment of additional macrophages to the atherosclerotic plaque.

In contrast to macrophages, VSMCs less frequently hybridized with MCP-1 probe. Individual VSMCs that hybridized strongly were sometimes surrounded by nonexpressing cells. Expression in these cells was not dependent on the proximity of tissue macrophages; hybridizing VSMCs were found in areas 
both with and without HAM56 staining. Because signals which stimulate MCP-1 production in VSMCs are not known and because MCP-1 production by VSMCs may be involved in the initial recruitment of macrophages to the vessel wall, we exposed cultured atheroma-derived VSMCs TNF $\alpha$ and $\gamma$ IFN, then determined MCP-1 mRNA levels by Northern blot. The increase in MCP- 1 expression induced by $\gamma$ IFN and supernatants of mixed lymphocyte culture suggest a possible role for activated $T$ lymphocytes in stimulation of vascular smooth muscle MCP-1 production.

The production of MCP-1 by MICs adds to the increasing evidence suggesting that these cells secrete a number of bioactive factors $(17,18)$. The origin of MICs and their role in atherogenesis remain unknown.

The identification of VSMCs and MICs as sources of MCP1 in atherosclerotic plaque suggests that this molecule may potentially play a role in monocyte infiltration in the early development of the plaque. Mediators stimulating monocyte infiltration into early fatty streaks are unknown, but these lesions have been identified as possible precursors to mature atherosclerotic plaques (3). It is tempting to speculate that MCP-1 production by VSMCs or MICs may be elicited by lipoproteins (11) or other stimuli in early atherosclerotic lesions. With infiltration of monocytes, a positive feedback mechanism might exist whereby infiltrating monocyte/macrophages secrete MCP-1 in large amounts, thereby attracting more peripheral blood monocytes to the developing atherosclerotic plaque. Antagonists of MCP-1 action will be needed to evaluate any causal role for MCP-1 in vivo.

In summary, we have shown that MCP-1 mRNA is expressed in atherosclerotic plaques in vivo. To our knowledge, this is the first study to demonstrate the production of a specific monocyte chemotactic agent in human atheroma.

\section{Acknowledgments}

We thank R. Stoney, W. Ehrenfeld, J. Goldstone, and J. Rapp of the Division of Vascular Surgery, University of California, San Francisco, D. E. Strandness of the Division of Vascular Surgery, University of Washington, for providing us with the tissues used in this study, and Dr. Lois Epstein of the University of California, San Francisco, for a generous gift of gamma interferon.

N. A. Nelken was supported by a grant from the Pacific Vascular Foundation and National Institutes of Health National Research Service Award grant F32 HL-08241. D. Gordon was supported by RO1 HL-42119. This work was supported in part by RO1 HL-43322.

\section{References}

1. Jonasson, L., J. Holm, O. Skalli, G. Bondjers, and G. K. Hannson. 1986. Regional accumulations of $\mathrm{T}$ cells, macrophages, and smooth muscle cells in the human atherosclerotic plaque. Arteriosclerosis. 6:131-138.

2. Gerrity, R. 1981. The role of the monocyte in atherogenesis. I. Transition of blood-borne monocytes into foam cells in fatty lesions. Am. J. Pathol. 103:181190.

3. Ross, R. 1986. The pathogenesis of atherosclerosis. N. Engl. J. Med. 314:488-500.

4. Robinson, E. A., T. Yoshimura, E. J. Leonard, S. Tanaka, P. R. Griffin, J. Shabanowitz, D. F. Hunt, and E. Appella. 1989. Complete amino acid sequence of a human monocyte chemoattractant, a putative mediator of cellular immune reactions. Proc. Natl. Acad. Sci. USA. 86:1850-1854.

5. Strieter, R. M., R. Wiggins, S. H. Phan, B. L. Wharram, H. J. Showell, D. G. Remick, S. W. Chensue, and S. L. Kunkel. 1989. Monocyte chemotactic protein gene expression by cytokine-treated human fibroblasts and endothelial cells. Biochem. Biophys. Res. Commun. 162:694-700.

6. Yoshimura, T., N. Yuhki, S. K. Moore, E. Appella, M. I. Lerman, and E. J. Leonard. 1989. Human monocyte chemoattractant protein-1 (MCP-1). Fulllength cDNA cloning, expression in mitogen-stimulated blood mononuclear leukocytes, and sequence similarity to mouse competence gene JE. FEBS (Fed. Eur. Biochem. Soc.) Lett. 244:487-493.

7. Kaczmarek, B., B. Calabretta, and R. Baserga. 1985. Expression of cell-cycle-dependent genes in phytohemagglytinin-stimulated human lymphocytes. Proc. Natl. Acad. Sci. USA. 82:5375-5379.

8. Rollins, B. J., T. Yoshimura, E. J. Leonard, and J. S. Pober. 1990. Cytokine-activated human endothelial cells synthesize and secrete a monocyte chemoattractant, MCP-1/JE. Am. J. Pathol. 136:1229-1233.

9. Valente, A. J., D. T. Graves, C. E. Vialle-Valentin, R. Delgado, and C. J. Schwartz. 1988. Purification of a monocyte chemotactic factor secreted by nonhuman primate vascular cells in culture. Biochemistry. 27:4162-4168.

10. Sica, A., J. M. Wang, F. Colotta, E. DeJana, A. Mantovani, J. J. Oppenheim, C. G. Larsen, C. O. C. Zachariae, and K. Matsushima. 1990. Monocyte chemotactic and activating factor gene expression induced in endothelial cells by IL-1 and tumor necrosis factor. J. Immunol. 144:3034-3038.

11. Berliner, J. A., M. C. Territo, A. Sevanian, S. Ramin, J. A. Kim, B. Bamshad, M. Esterson, and A. M. Fogelman. 1990. Minimally modified low density lipoprotein stimulates monocyte endothelial interactions. J. Clin. Invest. 85:1260-1266.

12. Cushing, S. D., J. A. Berliner, A. J. Valente, M. C. Territo, M. Navab, F Parhami, R. Gerrity, C. J. Schwartz, and A. M. Fogelman. 1990. Minimally modified low density lipoprotein induces monocyte chemotactic protein 1 in human endothelial cells and smooth muscle cells. Proc. Natl. Acad. Sci. USA. 87:5134-5138.

13. Furutani, Y., H. Nomura, M. Notake, Y. Oyamada, T. Fukui, M. Yamada, C. G. Larsen, J. J. Oppenheim, and K. Matsushima. 1989. Cloning and sequencing of the $\mathrm{CDNA}$ for human monocyte chemotactic and activating factor (MCAF). Biochem. Biophys. Res. Commun. 159:249-255.

14. Sambrook, J., E. F. Fritsch, and T. Maniatis. 1981. Molecular Cloning. A Laboratory Manual. Cold Spring Harbor Laboratory Press, Cold Spring Harbor, NY. 7.19-7.22.

15. Saiki, R. K., D. H. Gelfand, S. Stoffel, S. J. Scharf, R. Higuchi, G. T. Horn, K. B. Mullis, and H. A. Erlich. 1988. Primer-directed enzymatic amplification of DNA with a thermostable DNA polymerase. Science (Wash. DC). 233:10761078.

16. Sanger, F., S. Nicklen, and A. R. Coulson. 1977. DNA sequencing with chain-terminating inhibitors. Proc. Natl. Acad. Sci. USA. 74:5463-5469.

17. Wilcox, J. N., K. M. Smith, L. T. Williams, S. M. Schwartz, and D. Gordon. 1988. Platelet-derived growth factor mRNA detection in human atherosclerotic plaques by in situ hybridization. J. Clin. Invest. 82:1134-1143.

18. Wilcox, J. N., K. M. Smith, S. M. Schwartz, and D. Gordon. 1989. Localization of tissue factor in the normal vessel wall and in the atherosclerotic plaque. Proc. Natl. Acad. Sci. USA. 86:2839-2843.

19. Gown, A. W., T. Tsukada, and R. Ross. 1986. Human atherosclerosis. II. Immunocytochemical analysis of the cellular composition of human atherosclerotic lesions. Am. J. Pathol. 125:191-207.

20. Holthöfer, H., I. Virtanen, A. L. Karimiemi, M. Hormia, E. Linder, and A. Miettinen. 1982. Ulex europaeus I lectin as a marker for vascular endothelium in human tissues. Lab. Invest. 47:60-66.

21. Tsukada, T., D. Tippens, D. Gordon, and R. Ross. 1986. HHF35, a muscle-actin-specific monoclonal antibody. Am. J. Pathol. 126:51-60.

22. Terry, L. A., M. H. Brown, and P. C. L. Beverley. 1988. The monoclonal antibody UCHLl recognizes a $180 \mathrm{kD}$ component of the human leucocyte common antigen, CD45. Immunology. 64:331-339.

23. Libby, P., S. J. Warner, R. N. Salomon, and L. K. Birinyi. 1988. Production of platelet-derived growth factor-like mitogen by smooth-muscle cells from human atheroma. N. Engl. J. Med. 318:493-498.

24. Wilcox, J. N. 1991. Analysis of local gene expression in human atherosclerotic plaques by in situ hybridization. Trends Cardiovasc. Med. 1:17-24.

25. Wilcox, J. N., S. M. Schwartz, and D. Gordon. 1990. Local production of platelet-derived growth factor in the human atherosclerotic plaque. In Growth Factors, Differentiation Factors, and Cytokines. A. Habenicht, editor. SpringerVerlag, Berlin. 305-323. 